\title{
Effective Hamiltonians for Rapidly Driven Many-Body Lattice Systems: Induced Exchange Interactions and Density-Dependent Hoppings
}

\author{
A. P. Itin ${ }^{1,2}$ and M. I. Katsnelson ${ }^{1,3}$ \\ ${ }^{1}$ Radboud University, Institute for Molecules and Materials (IMM), Nijmegen 6525 AJ, Netherlands \\ ${ }^{2}$ Space Research Institute, Russian Academy of Sciences, Moscow 117997, Russia \\ ${ }^{3}$ Department of Theoretical Physics and Applied Mathematics, Ural Federal University, Ekaterinburg 620002, Russia
}

(Received 4 July 2014; published 13 August 2015)

\begin{abstract}
We consider 1D lattices described by Hubbard or Bose-Hubbard models, in the presence of periodic high-frequency perturbations, such as uniform ac force or modulation of hopping coefficients. Effective Hamiltonians for interacting particles are derived using an averaging method resembling classical canonical perturbation theory. As is known, a high-frequency force may renormalize hopping coefficients, causing interesting phenomena such as coherent destruction of tunneling and creation of artificial gauge fields. We find explicitly additional corrections to the effective Hamiltonians due to interactions, corresponding to nontrivial processes such as single-particle density-dependent tunneling, correlated pair hoppings, nearest neighbor interactions, etc. Some of these processes arise also in multiband lattice models, and are capable of giving rise to a rich variety of quantum phases. The apparent contradiction with other methods, e.g., Floquet-Magnus expansion, is explained. The results may be useful for designing effective Hamiltonian models in experiments with ultracold atoms, as well as in the field of ultrafast nonequilibrium magnetism. An example of manipulating exchange interaction in a Mott-Hubbard insulator is considered, where our corrections play an essential role.
\end{abstract}

DOI: 10.1103/PhysRevLett.115.075301

The idea of engineering effective Hamiltonians using high-frequency perturbations probably goes back to the famous Kapitza pendulum [1-4]. In classical and celestial mechanics there are many examples of systems with separation of typical time scales on slow and fast ones, and corresponding perturbation methods were developed a long time ago [5]. It is interesting to adopt these methods to the quantum realm, especially to lattice systems, where high-frequency perturbations are often used, e.g., for construction of quantum simulators (well-controllable quantum systems for simulating complicated condensed matter phenomena [6-8]).

Dynamical localization, transport, induced phase transitions have been studied in ultracold atoms in driven optical lattices [9-18] and in solid-state systems [19-27]. Most of the applications are based on modifying singleparticle hoppings by a high-frequency force, a phenomenon that is derived by averaging a Hamiltonian of the system (i.e., keeping only zeroth order terms in $1 / \omega$ (inverse frequency of perturbation) and neglecting all higher-order terms. However, for many realistic applications of such type, it is important to derive accurate effective Hamiltonians taking into account higher-order terms (see, e.g., Ref. [28]). Here we determine explicitly higher-order corrections to effective Hamiltonians of driven quantum lattices by elaborating a method [29-31] inspired by canonical perturbation theory. The corrections correspond to nontrivial (many-body) processes such as single-particle density-dependent tunneling,
PACS numbers: 67.85.-d, 03.65.Sq, 05.45.-a, 05.50.+q

correlated pair hoppings, nonlocal (extended) pair hopping, and so on. Using a suitable driving, we are able to suppress or enhance a particular process in the effective Hamiltonian. Such approach can be very useful for engineering particular Hamiltonians, for simulating solid-state phenomena via optical means, for accurate interpretation of experiments with driven lattice systems, etc. A particular application to a recent insightful proposal of ultrafast and reversible control of exchange interactions [32] is demonstrated.

The method can be seen either as a modification of the method of Ref. [31], or as a modification of the Magnus expansion approach [33,34]. Surprisingly, they can lead to different results. Correspondingly, some related methods available in the literature allow us to obtain results that are in accord with ours [28,31], while others [33,35] may lead to apparently different Hamiltonians. Especially, the difference between our approach and modifications of the Magnus expansion method [33] is important. Magnus expansion $[33,34]$ is a popular tool in physics and mathematics with a rapidly growing number of applications. The subtle issue is the order at which one can truncate the asymptotic expansion; as we show below and in Ref. [36], the first-order corrections obtained in the latter methods often do not make a contribution to the spectrum of the effective Hamiltonian in the same order. That is, physically meaningful corrections to the effective Hamiltonian appear only in the next, higher orders. To clarify our approach, let us first start with the Schrödinger equation for a lattice 
system (e.g., a particle in a driven tight-binding model), written in the matrix form

$$
i \dot{X}=\epsilon \mathcal{H} X \text {, }
$$

where $\epsilon=1 / \omega$ is a small parameter, $\omega$ is a frequency of perturbation, $\mathcal{H}(t)$ is the Hamiltonian of the system, $X$ is a column of coefficients of expansion of a quantum state in a certain basis, and fast time was introduced $(t \rightarrow t / \epsilon)$, resulting in the small coefficient $\epsilon$ in front of the rhs of Eq. (1), to put high-frequency dependence of the Hamiltonian in explicit form. Note that later we consider the case where a certain part of the Hamiltonian $H$ is large (of order of $\omega$ ), so that the rhs of Eq. (1) would contain contributions of order of 1 .

We may consider Eq. (1) as a classical dynamical system for a vector $X$. We adopt then a classical averaging method $[5,29,40-42]$ to this system, in such a way that allows convenient generalization to many-body systems [29]. One makes a unitary transformation $X=C \tilde{X}$ so that

$$
i \dot{\tilde{X}}=\left[C^{-1} \epsilon \mathcal{H} C-i C^{-1} \dot{C}\right] \tilde{X} .
$$

The expression in brackets is the new Hamiltonian. To get an effective time-independent Hamiltonian, the transformation is sought in the form $C=\exp \left[\epsilon K_{1}+\epsilon^{2} K_{2}+\epsilon^{3} K_{3}+\cdots\right]$, where $K_{i}$ are skew-Hermitian time-periodic matrices (with zero mean), which remove time-dependent terms from the Hamiltonian, leaving only time-independent ones. An iterative procedure analogous to the Hamiltonian averaging method [29] gives us

$$
\begin{aligned}
i \dot{K}_{1} & =\mathcal{H}(t)-\langle\mathcal{H}(t)\rangle \equiv\{\mathcal{H}\}, i K_{1}=\int\{\mathcal{H}\} d t, \\
i \dot{K}_{2} & =\left\{\mathcal{H} K_{1}-K_{1} \mathcal{H}-\frac{i}{2}\left(\dot{K}_{1} K_{1}-K_{1} \dot{K}_{1}\right)\right\}, \\
\epsilon \mathcal{H}_{\text {eff }} & =\left[C^{-1} \epsilon \mathcal{H} C-i C^{-1} \dot{C}\right]=\epsilon \mathcal{H}_{0}+\epsilon^{2} \mathcal{H}_{1}+\cdots,
\end{aligned}
$$

where curly brackets denote the time-periodic part of a timedependent function: $\{X\} \equiv X-\langle X(t)\rangle$, where $\langle X(t)\rangle \equiv$ $(1 / 2 \pi) \int_{0}^{2 \pi} X\left(t^{\prime}\right) d t^{\prime}$. Indefinite integrals above are defined up to an additive constant, which is chosen in such a way that $\left\langle K_{i}\right\rangle=0$. We have

$$
\begin{aligned}
& \mathcal{H}_{0}=\langle\mathcal{H}\rangle, \quad \mathcal{H}_{1}=\frac{1}{2}\left\langle\left[\{\mathcal{H}\}, K_{1}\right]\right\rangle, \\
& \mathcal{H}_{2}=\frac{1}{2}\left\langle\left[\{\mathcal{H}\}, K_{2}\right]\right\rangle+\frac{1}{12}\left\langle\left[\left\{\left[\{\mathcal{H}\}, K_{1}\right]\right\}, K_{1}\right]\right\rangle, \ldots
\end{aligned}
$$

where square brackets denote matrix commutation: $[A, B]=A B-B A$. This procedure resembles FloquetMagnus expansion [33]; however, there is an important difference due to lifting the unnecessary requirement $K_{i}(0)=0$ present in that method. As detailed in
Ref. [36], it often allows us to remove the correction $\mathcal{H}_{1}$, therefore obtaining much more accurate effective Hamiltonians, where corrections to $\mathcal{H}_{0}$ in the expansion start from $\mathcal{H}_{2}$.

Equation (3) allows us to consider, e.g., a particle in driven tight-binding models with various external potentials (see Refs. [29] and [36]). The general equations, Eq. (3), (which are in agreement with method of Ref. [30,31]) are also convenient for studying many-body lattice systems. Indeed, in the case of many particles, one can construct a corresponding Hamiltonian matrix and fulfil the same transformations. It is not necessary to consider Hamiltonians [Eq. (3)] in the matrix representation: one can use creation and annihilation operators. Indeed, consider now a 1D Bose-Hubbard model with a strong high-frequency driving:

$$
\begin{aligned}
H & =H_{\mathrm{BH}}+H_{d}(t), \quad H_{d}(t)=\omega \mathcal{E}(\omega t) \sum_{j} j n_{j}, \\
H_{\mathrm{BH}} & =J \sum_{i}\left(c_{i}^{\dagger} c_{i+1}+c_{i+1}^{\dagger} c_{i}\right)+U \sum_{i} n_{i}\left(n_{i}-1\right),
\end{aligned}
$$

where $J$ is the hopping parameter, $U$ is the interaction strength, and $c^{\dagger}, c$ are bosonic creation or annihilation operators. Following the approach we used in the singleparticle case, we make a preliminary transformation $U^{(0)}(t)=\exp \left[i f(\omega t) \sum_{j} n_{j}\right], \quad f(\omega t) \equiv \int_{0}^{t} \omega \mathcal{E}\left(\omega t^{\prime}\right) d t^{\prime}$, and make the rescaling of time, $t^{\prime}=\omega t=t / \epsilon$, so that the new Hamiltonian is $\epsilon \mathcal{H}(t)=\epsilon\left[\mathcal{H}_{0}+\delta H(t)\right]$, where

$$
\begin{aligned}
\mathcal{H}_{0} & =\sum_{i} J_{\mathrm{eff}}\left(c_{i}^{\dagger} c_{i+1}+c_{i+1}^{\dagger} c_{i}\right)+U \sum_{i} n_{i}\left(n_{i}-1\right), \\
\delta H(t) & =\sum_{i}\left[\delta^{+}(t) c_{i}^{\dagger} c_{i+1}+\delta^{-}(t) c_{i+1}^{\dagger} c_{i}\right], \\
J_{\mathrm{eff}} & =J\left\langle e^{i f(t)}\right\rangle, \quad \delta^{ \pm}(t)=J\left[e^{ \pm i f(t)}-\left\langle e^{i f(t)}\right\rangle\right] .
\end{aligned}
$$

In other words, the new Hamiltonian is $\epsilon \mathcal{H}(t)=$ $\epsilon\left[\sum_{i}\left(\delta_{0}^{+}(t) c_{i}^{\dagger} c_{i+1}+\delta_{0}^{-}(t) c_{i+1}^{\dagger} c_{i}\right)+U \sum_{i} n_{i}\left(n_{i}-1\right)\right]$, where $\delta_{0}^{ \pm}=J e^{ \pm i f(t)}$.

To find operators $K_{1}, K_{2}, \mathcal{H}_{1}, \mathcal{H}_{2}$ explicitly, we fulfil more complicated many-body calculations. In the first order, $\dot{K}_{1}=-i\{\mathcal{H}\}=-i \epsilon \sum_{i}\left[\delta^{+}(t) c_{i}^{\dagger} c_{i+1}+\delta^{-}(t) c_{i+1}^{\dagger} c_{i}\right]$, and therefore $K_{1}=-i \epsilon \sum_{i}\left[\delta_{1}^{+}(t) c_{i}^{\dagger} c_{i+1}+\delta_{1}^{-}(t) c_{i+1}^{\dagger} c_{i}\right]$, $\delta_{1}^{ \pm}=\int \delta^{ \pm}\left(t^{\prime}\right) d t^{\prime} . \quad$ Commutators $\quad\left[\mathcal{H}, K_{1}\right],\left[\left[\mathcal{H}, K_{1}\right], K_{1}\right]$, etc, are derived in Ref. [36]. The first-order terms in the new Hamiltonian (before averaging) are $\left[\mathcal{H}, K_{1}\right]=$ $-2 \epsilon i U \sum_{j} \delta_{1}^{+} c_{j}^{\dagger}\left(n_{j}-n_{j+1}\right) c_{j+1}-\delta_{1}^{-} c_{j+1}^{\dagger}\left(n_{j}-n_{j+1}\right) c_{j}$. These terms resemble somehow the result of the calculations of Ref. [35] (the structure of Hamiltonian is the same, but the coefficients $\delta_{1}^{ \pm}$are different). However, in our approach this contribution disappears during time-averaging (being averaged to zero: $\mathcal{H}_{1}=\frac{1}{2}\left\langle\left[\{\mathcal{H}\}, K_{1}\right]\right\rangle=0$ ), and therefore to find the nonvanishing contribution to the effective 
Hamiltonian, we need to consider the next orders of perturbation, exactly as in the single-particle case. This gives us (see Ref. [36])

$$
\mathcal{H}_{2}=-2 U\left(\Delta^{+} \hat{a}_{1}+\text { H.c. }\right)-2 U \Delta_{0} \hat{a}_{2},
$$

where

$$
\begin{aligned}
\Delta^{+} & =\frac{1}{2}\left\langle\delta_{2}^{+} \delta^{+}\right\rangle, \quad \Delta_{0}=\frac{1}{2}\left\langle\delta^{+} \delta_{2}^{-}+\delta_{2}^{+} \delta^{-}\right\rangle, \\
\hat{a}_{1} & =\sum_{j}\left(c_{j-1}^{\dagger}\left(4 n_{j}-n_{j+1}-n_{j-1}\right) c_{j+1}-2 c_{j}^{\dagger} c_{j}^{\dagger} c_{j+1} c_{j+1}\right), \\
\delta_{2}^{ \pm} & =\int \delta_{1}^{ \pm}\left(t^{\prime}\right) d t^{\prime}, \\
\hat{a}_{2} & =\sum_{j}\left[4 n_{j} n_{j+1}-2 n_{j}\left(n_{j}-1\right)-\left(c_{j-1}^{\dagger} c_{j+1}^{\dagger} c_{j}^{2}+\text { H.c. }\right)\right] .
\end{aligned}
$$

The effective Hamiltonian contains nearest-neighbor interactions and several types of correlated tunneling processes (pair tunneling $c_{j}^{\dagger} c_{j}^{\dagger} c_{j+1} c_{j+1}$ and pair "dissociation" or "association" process $\left.c_{j-1}^{\dagger} c_{j+1}^{\dagger} c_{j} c_{j} / c_{j}^{\dagger} c_{j}^{\dagger} c_{j+1} c_{j-1}\right)$. Because of these terms, one may expect a rich phase diagram of the driven system. In particular, it is known that the extended Bose-Hubbard model possesses a supersolid phase.

We note that in the case of harmonic perturbation $\mathcal{E}=-\mathcal{E}_{0} \sin t$, we have $\Delta^{+}=-\sum_{k=1}^{\infty}(-1)^{k}\left[J_{k}^{2}\left(\mathcal{E}_{0}\right) / k^{2}\right]$. This is a decaying oscillatory function of $\mathcal{E}_{0}$ which can be either positive or negative, and by varying the amplitude $\mathcal{E}_{0}$ one can either maximize its absolute value (e.g., at $\mathcal{E}_{0}=1.77$ ), or put it to zero (e.g., at $\mathcal{E}_{0}=3.33$ ). At the same time, $\Delta_{0}=-\sum_{k=1}^{\infty}\left[J_{k}^{2}\left(\mathcal{E}_{0}\right) / k^{2}\right]$, which is a nonvanishing oscillatory function of $\mathcal{E}_{0}$ with local minima at $\mathcal{E}_{0}=1.93,5.32$, etc. We see that using a suitable driving, it is possible to suppress or enhance particular processes in the effective Hamiltonian.

It is worth it to consider a classical limit $n_{i} \gg 1$, where one gets a driven discrete nonlinear Schrödinger equation (DNLSE). Applying canonical perturbation theory to the driven DNLSE we get an effective Hamiltonian which is indeed the classical limit of Eq. (6); i.e., it can be obtained from Eq. (6) by replacing operators with $c$ - numbers [36].

Consider now the driven Hubbard model

$$
\begin{aligned}
H & =H_{H}+H_{d}(t), \\
H_{d}(t) & =\omega \mathcal{E}(\omega t) \sum_{j} j n_{j}, \\
H_{H} & =J \sum_{i, \sigma}\left(c_{i, \sigma}^{\dagger} c_{i+1, \sigma}+c_{i+1, \sigma}^{\dagger} c_{i, \sigma}\right)+U \sum_{i} n_{i, \sigma} n_{i,-\sigma}
\end{aligned}
$$

(with $c_{i, \sigma}^{\dagger}, c_{i, \sigma}^{\dagger}$ being fermionic creation and annihilation operators) which becomes, after the preliminary transformation discussed above,

$$
\mathcal{H}=\sum_{i, \sigma}\left(\delta_{0}^{+} c_{i, \sigma}^{\dagger} c_{i+1, \sigma}+\delta_{0}^{-} c_{i+1, \sigma}^{\dagger} c_{i, \sigma}\right)+U \sum_{i} n_{i, \sigma} n_{i,-\sigma} .
$$

Calculations analogous to the Bose-Hubbard model case give us (see Supplemental Material [36])

$$
\begin{aligned}
\mathcal{H}_{1}= & 0, \\
\mathcal{H}_{2}= & U \Delta_{0}\left(-2 \mathcal{S}+\mathcal{A}+\mathcal{A}^{\dagger}+4 \mathbf{V}-2 V\right) \\
& +U\left[\Delta^{-}\left(4 \mathbf{R}-2 \mathcal{R}+R_{2}\right)+\text { H.c. }\right],
\end{aligned}
$$

where $\Delta^{-}=\left(\Delta^{+}\right)^{*}$, and several types of tunneling and interaction processes were denoted as $\mathcal{S}, A, R, \mathbf{R}, R_{2}, V$, and $\mathbf{V}$. $\mathcal{S}$ is the kinetic exchange interaction. $\mathcal{A}^{\dagger}$ denotes a tunneling process where a pair of atoms at the $j$ th site is dissociated into two atoms at neighboring sites $(j-1$ and $j+1)$. $\mathcal{A}$ denotes a corresponding correlated tunneling process of association of two atoms into a pair. $\mathcal{R}\left(\mathcal{R}^{\dagger}\right)$ denotes a correlated tunneling process where an extended pair of atoms tunnels to the right (left) neighboring sites. $\mathbf{R}\left(\mathbf{R}^{\dagger}\right)$ denotes tunneling of a localized pair of atoms to the right (left). $R_{2}\left(R_{2}^{\dagger}\right)$ denotes single-particle next-nearestneighbor density-dependent tunneling to the right (left); $V$ is the nearest-neighbor interaction, and $\mathbf{V}$ is the usual local interaction.

$$
\begin{aligned}
\mathcal{S} & =\sum_{j, \sigma} c_{j+1, \sigma}^{\dagger} c_{j, \sigma} c_{j,-\sigma}^{\dagger} c_{j+1,-\sigma}, \\
\mathcal{A} & =\mathcal{A}_{\uparrow, \downarrow}+\mathcal{A}_{\downarrow, \uparrow}, \quad \mathcal{A}_{\sigma,-\sigma}=\sum_{j} c_{j, \sigma}^{\dagger} c_{j-1, \sigma} c_{j,-\sigma}^{\dagger} c_{j+1,-\sigma}, \\
\mathcal{R} & =\mathcal{R}_{\uparrow \downarrow}+\mathcal{R}_{\downarrow \uparrow}, \quad \mathcal{R}_{\sigma,-\sigma}=\sum_{j} c_{j, \sigma}^{\dagger} c_{j-1, \sigma} c_{j+1,-\sigma}^{\dagger} c_{j,-\sigma}, \\
\mathbf{R} & =\sum_{j} c_{j+1, \downarrow}^{\dagger} c_{j, \downarrow} c_{j+1, \uparrow}^{\dagger} c_{j, \uparrow}, \\
R_{2} & =\sum_{j, \sigma} c_{j+1, \sigma}^{\dagger} c_{j-1, \sigma}\left(n_{j-1,-\sigma}-2 n_{j,-\sigma}+n_{j+1,-\sigma}\right), \\
V & =\sum_{j, \sigma} n_{j, \sigma} n_{j+1,-\sigma}, \quad \mathbf{V}=\sum_{j} n_{j, \downarrow} n_{j, \uparrow} .
\end{aligned}
$$

After returning to the physical time (remember that we did rescaling $t \rightarrow t / \epsilon)$, the second-order correction is $\left(1 / \omega^{2}\right) \mathcal{H}_{2}$ [Eq. (9)] [43].

The second-order corrections we found enter the effective Hamiltonian with a prefactor $U J^{2} / \omega^{2}$. We therefore formally require not only $\omega \gg J$, but also $\left(U J^{2} / \omega^{2}\right) \ll 1$ for our theory be applicable. Surprisingly, it works well even beyond these restrictions (Fig. 1). It is clear that the larger $U$ makes the corrections more important. Moreover, it is possible to find situations where our "corrections" drastically change effective low-energy Hamiltonians. For example, for $U \gg 1$ and half-filling, the Hubbard model describes a Mott insulator where remaining spin degrees of 
freedom are coupled by an antiferromagnetic exchange interaction $J_{\mathrm{ex}}=\left(2 J^{2} / U\right)$ (this is captured already in a two-site Hubbard model: among the four states (for total $S_{z}=0$ ), the two low-lying states are singlet and triplet states with one electron per site and energies $E_{S}=-\left(4 J^{2} / U\right), E_{T}=0$. The spectrum at low energies is described by a spin Hamiltonian $2 J_{\mathrm{ex}} \mathbf{s}_{1} \mathbf{s}_{2}$ with $J_{\mathrm{ex}}=$ $\left.\left(E_{T}-E_{S}\right) / 2=\left(2 J^{2} / U\right)\right)$. If parameters in the driven Hubbard model are such that $\left(J^{2} / U\right) \sim U\left(J^{2} / \omega^{2}\right)$, i.e., $U \sim \omega$, then high-frequency corrections are of the same order as the effective exchange interaction itself, and therefore play a crucial role. Very recently, it was proposed to control exchange interactions by time-periodic modulation of an electric field [32]. The corresponding results of Ref. [32] can be reproduced and enlightened in our approach. For the two-site model, the correction to the effective Hamiltonian simplifies to $A\left(\mathcal{E}_{0}\right)\left[\left(n_{1 \downarrow}-\right.\right.$ $\left.\left.n_{2 \downarrow}\right)\left(n_{1 \uparrow}-n_{2 \uparrow}\right)-\left(c_{2 \uparrow}^{\dagger} c_{1 \uparrow} c_{1 \downarrow}^{\dagger} c_{2 \downarrow}+c_{2 \downarrow}^{\dagger} c_{1 \downarrow} c_{1 \uparrow}^{\dagger} c_{2 \uparrow}\right)\right]-C\left(\mathcal{E}_{0}\right)$ $\left(d_{2}^{\dagger} d_{1}+\right.$ H.c. $)$, where $d_{k}$ are annihilation operators of pairs (doublons), $A$ and $C$ are functions of driving that in the case of harmonic driving are equal to $-\left(4 U J^{2} / \omega^{2}\right) \sum_{1}^{\infty}\left[J_{m}^{2}\left(\mathcal{E}_{0}\right) / m^{2}\right]+O\left(1 / \omega^{4}\right)$ and $\left(4 U J^{2} /\right.$ $\left.\omega^{2}\right) \sum_{1}^{\infty}\left[(-1)^{m} J_{m}^{2}\left(\mathcal{E}_{0}\right) / m^{2}\right]+O\left(1 / \omega^{4}\right)$, correspondingly. Parameter $A$ (which gives the strength of exchange interaction) can be calculated taking into account an infinite number of terms in $1 / \omega$ expansion, in case we leave only the leading order contribution in $U$ in every term. Then, $A=-4 J^{2} U \sum_{m=1}^{\infty} J_{m}^{2}\left(\mathcal{E}_{0}\right) /\left[m^{2} \omega^{2}-U^{2}\right]$. It is interesting that this result, obtained under assumption $\omega>U$, remains valid also for $\omega<U$ [32]. We can obtain an even more accurate expression for the effective interaction, which includes influence of other terms in the effective Hamiltonian: eigenvalues of the effective two-site model can be obtained analytically (see Supplemental Material [36]). The half-distance between the two lowest levels is equal to $\Delta E / 2 \equiv J_{\mathrm{ex}}=\frac{1}{4}(A+C-U+$ $\left.\sqrt{16 J_{e}^{2}+(U+3 A-C)^{2}}\right) \approx\left(2 J_{e}^{2} / U\right)+A+\left(2 J_{e}^{2} / U^{2}\right)(C-$ $3 A)+\cdots$ For parameters used in Ref. [32], $J_{\text {ex }}$ is shown on Fig. 1. It reproduces calculations of Ref. [32] amazingly well (see Fig. 1 and Ref. [36]). Moreover, it shows that our second order corrections provide reliable approximation (unless tunneling is completely suppressed by driving), and therefore for longer lattices, our effective Hamiltonian adequately describes modification of effective exchange interaction, as well as emergence of auxiliary terms. Indeed, for large $U$, one can see that the most important contribution to the effective Hamiltonian comes from $\mathcal{S}$, other terms increase the number of double occupancies and therefore are suppressed by the (original) interaction term of the Hubbard model. One can eliminate them via a standard approach, by a canonical transformation which renormalizes coefficients of the remaining terms, i.e., exchange interaction [45]. When $J^{2} / U \sim U\left(J^{2} / \omega^{2}\right)$, with a good accuracy we find the effective exchange interaction

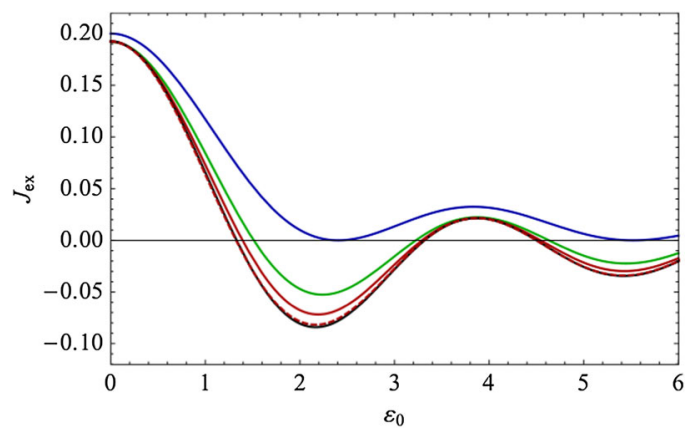

FIG. 1 (color online). Exchange interaction $J_{\text {ex }}$ in the driven fermionic two-site model as a function of the driving strength $\mathcal{E}_{0}$. Parameters: $U=10$, "bare" tunneling $J=-1, \omega=16$. Harmonic driving changes the effective tunneling constant $\left(J \rightarrow J_{e}=\right.$ $\left.J J_{0}\left(\mathcal{E}_{0}\right)\right)$ affecting the exchange interaction in the zeroth order in $1 / \omega: J_{\mathrm{ex}}^{(0)}=\left[2 J_{e}^{2}\left(\mathcal{E}_{0}\right) / U\right]$. Solid curves, from up to down: bare exchange interaction $J_{\mathrm{ex}}^{(0)}$; theoretical prediction $J_{\mathrm{ex}}^{(2)}$ with corrections up to the second order in $1 / \omega$ taken into account; $J_{\mathrm{ex}}^{(4)}$ with corrections up to the fourth order in $1 / \omega$ taken into account; $J_{\mathrm{ex}}^{(\infty)}$ with infinite order of terms in $1 / \omega$ expansion taken into account (with leading order in $U$ contribution in each term) (the lowest solid curve). Dashed curve (nearly indiscernible from the lowest solid curve): numerical value of the exchange interaction (kindly provided by $\mathrm{J}$. Mentink). In the regions where $J_{e}$ is strongly suppressed by driving (around zeros of $J_{\mathrm{ex}}^{(0)}$ ), the second order corrections become insufficient, and one needs to include fourth and higher orders in $1 / \omega . J_{\mathrm{ex}}^{(2),(4), \ldots}$ are defined as the half-distance between the two lowest levels of the effective Hamiltonian of the 2-site model [36].

is composed of the usual $J^{2} / U$ part and our correction from $\mathcal{S}$. So the driving not only modifies the $J^{2} / U$ part (by renormalizing $J \rightarrow J_{e}$ ), but also adds a "correction" $\mathcal{S}$ which can be of the same order. Suppression of the other terms in the effective Hamiltonian at large $U$ justify usage of the two-site model result in the extended system: we see that at least in the $1 \mathrm{D}$ case, the change of the exchange interaction is nearly the same as in the two-site system. Recently, the fermionic two-site system was realized experimentally [46]. For a long 1D lattice, the ability to switch the exchange interaction to the ferromagnetic type implies an interesting possibility of simulating an unusual spin-polaron excitation in driven lattices: a bound state of an extra fermion and a magnon [47]. Such a quasiparticle has peculiar properties, e.g., large effective mass [47], [36]. Experimental and theoretical studies of polarons and spin-polarons in ultracold gases is currently a very active topic of research [48]. One can also switch on and off single-particle and doublon hopping by varying parameters of driving [36].

To formalize the intriguing accuracy of summation of $U$ terms in the high-frequency expansion, we propose a modified version of the averaging method specifically suited for the large- $U$ case. Let us separate the $U$-dependent part of the Hamiltonian $\mathcal{H}_{U}$, and consider in detail the case $\omega=O(U)$. After introducing the fast time, our system has a form different from that of Eq. (1) 


$$
i \dot{X}=\left[\mathcal{H}_{\epsilon U}+\epsilon \mathcal{H}(t)\right] X,
$$

where $\mathcal{H}_{\epsilon U}$ is of the order of 1 . We have the system where driving is fast with respect to all time scales except $U$. We avoid the case of exact resonances $U=m \omega$. Considering Eq. (11), we get modified equations for the transformation $C$ and the effective Hamiltonian. After returning to the original time,

$$
\begin{aligned}
\mathcal{H}_{\text {eff }}= & \mathcal{H}_{U}+\langle\mathcal{H}\rangle+\epsilon \mathcal{H}_{1}+\epsilon^{2} \mathcal{H}_{2}+\cdots, \\
\mathcal{H}_{1}= & \frac{1}{2}\left\langle\left[\{\mathcal{H}\}, K_{1}\right]\right\rangle=\frac{1}{2} \sum_{m \neq 0}\left[\mathcal{H}^{(m)}, K_{1}^{(-m)}\right], \\
\mathcal{H}_{2}= & \frac{1}{2}\left\langle\left[\{\mathcal{H}\}, K_{2}\right]\right\rangle+\frac{1}{12}\left\langle\left[\left[\{\mathcal{H}\}, K_{1}\right], K_{1}\right]\right\rangle \\
& +\frac{1}{2}\left\langle\left[\left[H_{\epsilon U}, K_{2}\right], K_{1}\right]\right\rangle,
\end{aligned}
$$

where $\quad K_{1} \equiv \sum K_{1}^{(m)} \exp (i m t), K_{2} \equiv \sum K_{2}^{(m)} \exp (i m t)$ are now should be determined from equations for the Fourier harmonics $K_{1}^{(m)}, K_{2}^{(m)}$

$$
\begin{aligned}
K_{1}^{(m)} & =-\frac{1}{m}\left(\mathcal{H}^{(m)}+\left[\mathcal{H}_{\epsilon U}, K_{1}^{(m)}\right]\right) \equiv R_{m}\left(\mathcal{H}^{(m)}\right), \\
K_{2}^{(m)} & =-\frac{1}{m}\left(\Phi^{(m)}+\left[\mathcal{H}_{\epsilon U}, K_{1}^{(m)}\right]\right) \equiv R_{m}\left(\Phi^{(m)}\right), \\
\Phi^{(m)} & =\left[\langle H\rangle, K_{1}^{(m)}\right]+\frac{1}{2} \sum_{k+l=m}\left[\mathcal{H}^{(m)}, K_{1}^{(l)}\right] .
\end{aligned}
$$

Solving these equations for the two-site model, we get

$$
\mathcal{H}_{U}+\langle H\rangle+\epsilon \mathcal{H}_{1}=\left(\begin{array}{cccc}
-A & -A & J_{e} & J_{e} \\
-A & -A & J_{e} & J_{e} \\
J_{e} & J_{e} & U+A & -C \\
J_{e} & J_{e} & -C & U+A
\end{array}\right),
$$

where for the case of harmonic driving $A=$ $-\sum_{m \neq 0}\left[2 J_{m}\left(\mathcal{E}_{0}\right)^{2} \epsilon^{2} U / m^{2}-(\epsilon U)^{2}\right], C=\sum_{m \neq 0}\left[2 J_{m}\left(\mathcal{E}_{0}\right) J_{-m}\right.$ $\left.\left(\mathcal{E}_{0}\right) \epsilon^{2} U / m^{2}-(\epsilon U)^{2}\right]$. It is interesting that in this modification (developed for the case $\omega \sim U \gg J$ ), by summing the first 3 terms in the expansion, we get the result which coincides with summation of infinite number of terms in our initial approach. The case $\omega<U$ is very interesting since it allows us to amplify antiferromagnetic exchange interaction (in the opposite case $\omega>U$ exchange interaction is always diminished). This amplification is interesting, e.g., from the point of view of recent experiments with ultracold fermionic atoms [49], where short-range quantum magnetism was achieved by loading atoms in dimerized lattices and then merging the plaquettes. Amplification of antiferromagnetic interactions by driving can be easily combined with this method.

To conclude, a convenient method based on canonical transformations has been applied to two different lattice systems: driven Hubbard and Bose-Hubbard models. For a general high-frequency driving, we derive explicitly effective Hamiltonians including corrections from interactions. In the regime $\omega \gg U \sim J$, density dependent tunnelings play an important role in the effective Hamiltonian. Compared to other approaches (e.g., Ref. [35]), we reveal that physically meaningful contributions to the effective Hamiltonian are given by the second order corrections. While it is clear that effective Hamiltonians look differently in different frames, it was not clear that this requires a careful check of the order at which an effective Hamiltonian might be truncated. That is, expansion in small parameter $1 / \omega$ may give a nonvanishing first-order term, which one may assume to be a valid lowest order correction [35]. However, if this term can be completely removed by a canonical transformation, it means it does not contribute to the spectrum of the effective Hamiltonian in that order, and one needs to start consideration from the second order corrections. In present experiments with shaken optical lattices a frequency of driving is typically chosen in the $\mathrm{kHz}$ regime (much higher than time scales related to trapping potentials and (weak) interactions, and much lower than the interband transition frequency). Explicit knowledge of corrections from trapping potentials [29] and many-body interactions we derived here allow us to extend the range of the parameters of shaken lattice experiments to the sub-kHz regime. In the regime $(\omega \sim U \gg J)$, the corrections drastically influence the effective (Heisenberg) Hamiltonian, which is important for ultrafast control of magnetism in solids and simulation of magnetism with ultra cold atoms. In the latter case, not only amplification of antiferromagnetic exchange is possible, but also switching to ferromagnetic exchange interaction, which allows simulation of ferromagnetic $t J$ model. Another inspiring direction of applications is photo-induced superconductivity and metal-insulator transitions [25-27], where recent studies show the importance of induced Kapitza-like effective potentials [26].

We acknowledge financial support by the ERC (Project 338957 FEMTO/NANO) and by NWO via Spinoza Prize. We are grateful to A. I. Neishtadt, M. Eckstein, J. Mentink, A. Polkovnikov, A. Eckardt, S. Jochim, A. Lichtenshtein, A. Nocera, J. Simonet, M. Thorwart, and T. Rexin for inspiring discussions. Partial support from RFBR (Project No. 13-01-00251) is acknowledged.

Note added.-Recently, we learned about a relevant study [50] which supports our ideas from a different perspective.

[1] N. N. Bogolyubov, Sbornik trudov Instituta stroitel'noy mekhaniki AN USSR, 9 (1950); [Proc. Inst. Struct. Mech., 9 (1950)].

[2] P. L. Kapitza, Sov. Phys. JETP 21, 588 (1951). 
[3] A. Stephenson, Philos. Mag. 15, 233 (1908).

[4] Stabilization of unstable equilibrium of a pendulum with high-frequency driving was studied first in Ref. [3] using a linearization procedure, i.e., without introducing effective potentials (elaborated in Refs. [1,2]).

[5] V. I. Arnold, V. V. Kozlov, and A. I. Neishtadt, Mathematical Aspects of Classical and Celestial Mechanics 3rd ed (Springer, Berlin, 2006).

[6] R. Feynman, Int. J. Theor. Phys. 21, 467 (1982).

[7] J. I. Cirac and P. Zoller, Nat. Phys. 8, 264 (2012); I. Bloch, J. Dalibard, and S. Nascimbne, Nat. Phys. 8, 267 (2012).

[8] M. Lewenstein et al., Ultracold Atoms in Optical Lattices: Simulating Quantum Many-Body Systems (Oxford University Press, Oxford, 2012).

[9] H. Lignier, C. Sias, D. Ciampini, Y. Singh, A. Zenesini, O. Morsch, and E. Arimondo, Phys. Rev. Lett. 99, 220403 (2007).

[10] A. Eckardt, M. Holthaus, H. Lignier, A. Zenesini, D. Ciampini, O. Morsch, and E. Arimondo, Phys. Rev. A 79, 013611 (2009).

[11] J. Heinze, J. S. Krauser, N. Fläschner, B. Hundt, S. Götze, A. P. Itin, L. Mathey, K. Sengstock, and C. Becker, Phys. Rev. Lett. 110, 085302 (2013).

[12] J. Struck, C. Ölschläger, M. Weinberg, P. Hauke, J. Simonet, A. Eckardt, M. Lewenstein, K. Sengstock, and P. Windpassinger, Phys. Rev. Lett. 108, 225304 (2012).

[13] S. Greschner, G. Sun, D. Poletti, and L. Santos, Phys. Rev. Lett. 113, 215303 (2014); N. Goldman and J. Dalibard, Phys. Rev. X 4, 031027 (2014).

[14] J. Struck, C. Olschlager, R. Le Targat, P. Soltan-Panahi, A. Eckardt, M. Lewenstein, P. Windpassinger, and K. Sengstock, Science 333, 996 (2011).

[15] N. Strohmaier, Y. Takasu, K. Günter, R. Jördens, M. Köhl, H. Moritz, and T. Esslinger, Phys. Rev. Lett. 99, 220601 (2007); A. Alberti, V. V. Ivanov, G. M. Tino, and G. Ferrari, Nat. Phys. 5, 547 (2009); A. Zenesini, H. Lignier, D. Ciampini, O. Morsch, and E. Arimondo, Phys. Rev. Lett. 102, 100403 (2009); A. Eckardt, P. Hauke, P. Soltan-Panahi, C. Becker, K. Sengstock, and M. Lewenstein, Europhys. Lett. 89, 10010 (2010).

[16] K. Kudo, T. Boness, and T. S. Monteiro, Phys. Rev. A 80, 063409 (2009); K. Kudo and T. S. Monteiro, Phys. Rev. A 83, 053627 (2011); A. R. Kolovsky, E. A. Gomez, and H. J. Korsch, Phys. Rev. A 81, 025603 (2010).

[17] A. Eckardt, C. Weiss, and M. Holthaus, Phys. Rev. Lett. 95, 260404 (2005).

[18] A. Zenesini, H. Lignier, D. Ciampini, O. Morsch, and E. Arimondo, Phys. Rev. Lett. 102, 100403 (2009).

[19] D. H. Dunlap and V. M. Kenkre, Phys. Rev. B 34, 3625 (1986).

[20] M. Holthaus, Phys. Rev. Lett. 69, 351 (1992).

[21] M. Holthaus and D. W. Hohe, Philos. Mag. B 74, 105 (1996).

[22] M. Glück, A. R. Kolovsky, and H. J. Korsch, Phys. Rep. 366, 103 (2002).

[23] N. H. Lindner, G. Refael, and V. Galitski, Nat. Phys. 7, 490 (2011).

[24] T. Kitagawa, T. Oka, A. Brataas, L. Fu, and E. Demler, Phys. Rev. B 84, 235108 (2011); M. S. Rudner, N. H. Lindner, E. Berg, and M. Levin, Phys. Rev. X 3, 031005 (2013).

[25] M. Rini, R. Tobey, N. Dean, J. Itatani, Y. Tomioka, Y. Tokura, R. W. Schoenlein, and A. Cavalleri, Nature (London) 449, 72 (2007).
[26] A. Subedi, A. Cavalleri, and A. Georges, Phys. Rev. B 89, 220301(R) (2014).

[27] D. Fausti, R. I. Tobey, N. Dean, S. Kaiser, A. Dienst, M. C. Hoffmann, S. Pyon, T. Takayama, H. Takagi, and A. Cavalleri, Science 331, 189 (2011); S. Kaiser et al., Phys. Rev. B 89, 184516 (2014).

[28] S. Longhi, Phys. Rev. B 77, 195326 (2008).

[29] A. P. Itin and A. I. Neishtadt, Phys. Lett. A 378, 822 (2014).

[30] T. P. Grozdanov and M. J. Rakovic, Phys. Rev. A 38, 1739 (1988).

[31] S. Rahav, I. Gilary, and S. Fishman, Phys. Rev. A 68, 013820 (2003).

[32] J. H. Mentink, K. Balzer, and M. Eckstein, Nat. Commun. 6, 6708 (2015).

[33] S. Blanes, F. Casas, J. A. Oteo, and J. Ros, Phys. Rep. 470, 151 (2009).

[34] W. Magnus, Commun. Pure Appl. Math. 7, 649 (1954).

[35] A. Verdeny, A. Mielke, and F. Mintert, Phys. Rev. Lett. 111, 175301 (2013).

[36] See Supplemental Material at http://link.aps.org/ supplemental/10.1103/PhysRevLett.115.075301 for more technical details and examples, which includes Refs. [37-39].

[37] J. Spalek, Acta Phys. Polon. A 111, 409 (2007); J. Spalek and A. M. Oles, Physica (Amsterdam) B86, 375 (1977).

[38] G. I. Japaridze and E. Muller-Hartmann, Phys. Rev. B 61, 9019 (2000).

[39] M. I. Auslender, M. I. Katsnelson, and V. S. Vonsovsky, J. Magn. Magn. Mater. 15-18, 906 (1980).

[40] A. P. Itin, R. de la Llave, A. I. Neishtadt, and A. A. Vasiliev, Chaos 12, 1043 (2002); X. Leoncini, A. I. Neishtadt, and A. A. Vasiliev, Phys. Rev. E 79, 026213 (2009).

[41] A. P. Itin and A. I. Neishtadt, Phys. Rev. E 86, 016206 (2012).

[42] A. P. Itin et al. (to be published).

[43] Note that a two-particle problem in a driven asymmetric Hubbard model was recently considered in Ref. [44], where formation of a doublon of two fermions (an extended pair) residing on neighboring cites and cotunneling along the lattice was found (bare tunneling constants of the two fermions being different, $J_{\uparrow} \neq J_{\downarrow}$ ). The first-order correction found in Ref. [44] is proportional to $J_{\uparrow}-J_{\downarrow}$, i.e., it completely disappears in the symmetric limit $J_{\uparrow}=J_{\downarrow}$, in accordance with our findings.

[44] S. Longhi and G. DellaValle, Phys. Rev. A 87, 013634 (2013).

[45] A. H. MacDonald, S. M. Girvin, and D. Yoshioka, Phys. Rev. B 37, 9753 (1988).

[46] S. Murmann, A. Bergschneider, V. M. Klinkhamer, G. Zürn, T. Lompe, and S. Jochim, Phys. Rev. Lett. 114, 080402 (2015).

[47] M. I. Katsnelson, Fiz. Met. Metalloved. 54, 396 (1981).

[48] S. Nascimbene, N. Navon, K. J. Jiang, L. Tarruell, M. Teichmann, J. McKeever, F. Chevy, and C. Salomon, Phys. Rev. Lett. 103, 170402 (2009); A. Kantian, U. Schollwöck, and T. Giamarchi, Phys. Rev. Lett. 113, 070601 (2014); E. V. H. Doggen, A. Korolyuk, P. Törmä, and J. J. Kinnunen, Phys. Rev. A 89, 053621 (2014); P. A. Lee, N. Nagaosa, and X.-G. Wen, Rev. Mod. Phys. 78, 17 (2006).

[49] D.Greif, T. Uehlinger, G. Jotzu, L. Tarruell, and T. Esslinger, Science 340, 1307 (2013).

[50] A. Eckardt and E. Anisimovas, arXiv:1502.06477. 